\title{
Investigating Neural Substrates of Individual Independence and Interdependence Orientations via Efficiency-based Dynamic Functional Connectivity: A Machine Learning Approach
}

\author{
Yifan Zhu, Graduate Student Member, IEEE, Xuesong Li, Yang Sun, Haixu Wang, \\ Hua Guo, and Jie Sui
}

\begin{abstract}
The self-construal is one of the most significant cultural markers in humans. Accordingly, mapping the relationship between brain activity and self-construal contributes to understanding the nature of such psychological traits. Existing studies have mainly focused on static functional brain activities in specific brain regions. However, evidence has suggested that the functional connectivity of the brain network is dynamic over time and the high-level psychological processes might require collaboration among multiple regions. In the present study, we explored the dynamic connection patterns of the two most representative types of self-construal traits, namely independence and interdependence, using machine learning-based models. We performed resting-state functional MRI (rs-fMRI) on a sample of young adults $(n=359)$ who completed Singelis' Self-Construal Scale, and constructed the efficiency-based dynamic functional connectivity (FC) networks. XGBoost Regression was subsequently applied to learn the relationship between the dynamic FC and the two self-construals without any priori bias or hypothesis. The performance of the regression model was validated by the nested 10 -fold cross-validation. The results showed that the efficiency-based dynamic FC could identify the orientations of independence and interdependence. The comparison analyses revealed that prediction accuracy using this dynamic FC method was significantly improved compared to the conventional static FC method. By exploring key connectivities selected by the regression model, we observed that the independence orientation was mainly characterized by the righthemisphere FC, while the interdependence orientation by the lefthemisphere FC. The results suggest that the self-construals are associated with distributed neural networks the entire brain. These findings provide the pivotal ingredients toward the biological essence of culturally related variables in the brain by taking advances in cultural psychology, neuroscience, together with machine-learning analytic technologies.
\end{abstract}

Index Terms-self-construal, resting state functional connectivity (rsFC), nodal efficiency, dynamic functional connectivity

\section{INTRODUCTION}

$\mathrm{T}$ HE concept of self-construal establishes a system to provide perception, comprehension and interpretation on individuals and their relation to others in a social enviornment [1-4]. Markus et al. [1] firstly proposed to categorize people into independent self-construal (IndSC) and interdependent self-construal (InterSC) by examining whether they construe themselves as individuals and separate from others, or they are defined by relationships with others [5]. Generally, IndSC is associated with an emphasis on personal agency and uniqueness from others. In contrast, InterSC is associated with an emphasis on the relations to other people and with the maintenance of collective values, and pursuing social harmony. Although Markus et al. noted that these are only two of many possible self-construals, the term self-construal has become virtually synonymous with independence and interdependence [4]. Therefore, the identification of IndSC and InterSC becomes a typical cultural marker in humans. For decades, the concept of self-construal has attracted considerable attention in psychology and sociology [6-10].

Recent research in social neuroscience has verified that selfrelated processing is associated with brain activities located in multiple regions $[11,12]$. For example, researchers showed that self-construals are associated with multiple cognitive/affective neural processes, such as moderating associations between trait creativity and social brain network, affecting the functional organization of the human brain and behavior under different cultural backgrounds [3, 13, 14]. Other researchers also reported the brain modulation mechanisms for the orientations of independence and interdependence in other cognitive activities such as choice justification [15], reward [16], pain perception [17]. Specifically, from the perspective of fMRI, existing studies reported correlations between self-construals and task-based brain activities [18-21]. Although stimuli of these studies prompted the process of self-construal, different

H. Guo is with the Center for Biomedical Imaging Research, Department of Received in XX,XX,XXXX.

Y. Zhu, X. Li are with the School of Computer Science and Technology, Beijing Institute of Technology, Beijing, China.

Y. Sun is with the School of Educational Science, Shenyang Normal University, Shenyang, China.

H. Wang is with the Department of Psychology, Tsinghua University, Beijing, China.
Biomedical Engineering, School of Medicine, Tsinghua University, Beijing, China.

J. Sui is with the School of Psychology, University of Aberdeen, Aberdeen, UK.

Correspondence to: X. Li and J. Sui (e-mail: lixuesong@bit.edu.cn, jie.sui@abdn.ac.uk). 
protocols made the observed results not the same as each other. Besides, the task-driven approach also brings obstacles to the collection of large amounts of image data. On the other hand, existing resting state fMRI-based studies that investigate neural characteristics of self-construal are mainly based on specific regions of interest (ROI), while there is still little whole brainbased research [22]. To summarize, there is little work that explores the neural basis of the self-construal using resting-state whole-brain hodological (neural pathways) approach combined with topology (brain regions), although this approach is crucial for understanding the nature of social concepts [23-25]. In other words, the relationship between self-construals and wholebrain functional connectivity (FC) remains unclear.

Exploring FC has significantly contributed to our understanding of the brain's functional network architectures in health and disease [26]. Evidence shows that FC, associated with ongoing rhythmic activity, is dynamic instead of remaining stationary over time. In the recent fMRI studies, researchers found that many clinical symptoms and cognitive traits were encoded more evidently in dynamic functional connectome than conventional static counterpart [27-30]. Additionally, powered by emerging methods and models for spatial dynamic properties [31, 32], topological properties [29, 33 ], machine learning methods in neuroscience [34, 35] and validation scheme [36], whole-brain dynamics of connectivity or networks could be further investigated to establish stable generalized biomarkers (i.e. a traceable substance to examine self-construal measured by instruments) for a better understanding of the trait of self-construal. When brain regions located in different parts of the brain exhibit similar topological characteristics in the time dimension, this collaboration (or correlation) has potential implications for understanding these disorders and traits [37].

Building on these previous studies and advances, in the presented study, we applied machine learning-based regression models to investigate the self-construals (i.e. IndSC and InterSC) based on dynamic connectome-based brain networks of rs-fMRI data. According to the previous studies, the selfconstrual may be associated with a large scale of functional integrity of distributed networks and involve the mutual synchronization of multiple brain regions [13, 18-20, 38]. Thus, we assumed that the self-construals are encoded more explicitly in the dynamic FC than the static one, and utilized the efficiency attribute of the brain network to depict dynamic synchronization of different brain regions. To validate this hypothesis, we exploited XGBoost, a machine learning-based regression model without prior bias, to find the relationship between the efficiency-based dynamic FC and self-construals. Following the nested 10-fold cross-validation on the regression model, we compared the performance of using efficiency-based dynamic FC with other conventional approach to validate our hypothesis. By using this approach without prior bias, we further investigated significant dynamic functional connectivity weighted by the XGBoost model, thereby analyzing the connectome pattern for identifying independence and interdependence self-construals. Unbiased machine learning methods provide a propitious window to understanding social functions of the human brain that was previously underinvestigated. The present study therefore examined contributions of the proposed dynamic functional connectivity to self-construal, a social culture dimension of the self-concept, using machine learning approaches. Specifically, (1) Contrasting to the conventional static FC, we innovatively propose a sliding window-based dynamic correlation of nodal efficiency, namely efficiency-based dynamic FC, on whole brain resting-state functional connectivity ( $\mathrm{rsFC}$ ), to mine higher-order social information that can hardly be extracted from static rsFC; (2) We utilize the XGBoost regression model to learn the relationship between scores of individuals' IndSC as well as InterSC and the efficiency-based dynamic FC, then validate the performance of the model with nested 10-fold cross-validation, thereby demonstrating the benefit of using the proposed whole-brain dynamic rsFC without pre-defined bias; (3) We interpreted the patterns (regions and connections) weighted by the XGBoost model for identifying IndSC and InterSC, thereby further investigating the characteristic of hemisphere dominance for self-construal.

\section{MATERIALS AND METHODS}

\section{A. Participants}

359 undergraduate or graduate students (159 males; age: 1830, average: 22.74 ) were recruited. Written informed consents were obtained from all participants. The research protocol was approved by the Ethics Committee of School of Medicine in Tsinghua University. According to self-reported records, these participants had no life-time psychiatric disorder, no history of neurological disorders, and no gross abnormalities as confirmed using MRI. Participants were excluded if they had: (i) a history of substance, drug or alcohol dependence; (ii) observable brain abnormalities using MRI; (iii) metal devices such as electronic implants; (iv) the mean framewise displacement value exceeding $0.5 \mathrm{~mm}$, or the maximum displacement caused by head motion larger than $1 \mathrm{~mm}$. Eventually, by excluding 36 participants due to the head motion, a dataset including 323 participants (displacement: mean $=0.21 \pm 0.12 \mathrm{~mm}$ ) was derived for the following investigation. The code utilized in the following experiments is available at GitHub ${ }^{1}$.

\section{B. Assessment of IndSC and InterSC}

The degree of individual self-construal was evaluated using the Singelis' Self-Construal Scale (SCS) [39] with Chinese translation. The Singelis' Self-Construal Scale has 30 items, half of which measure IndSC (e.g. "I do my own thing, regardless of what others think.") while the other half measure InterSC (e.g. "I will sacrifice my self-interest for the benefit of the group I am in."). Participants are required to rate the extent to which they agreed with. Each item uses a 7-option Likertlike scale from 1 (strongly disagree) to 7 (strongly agree). In 

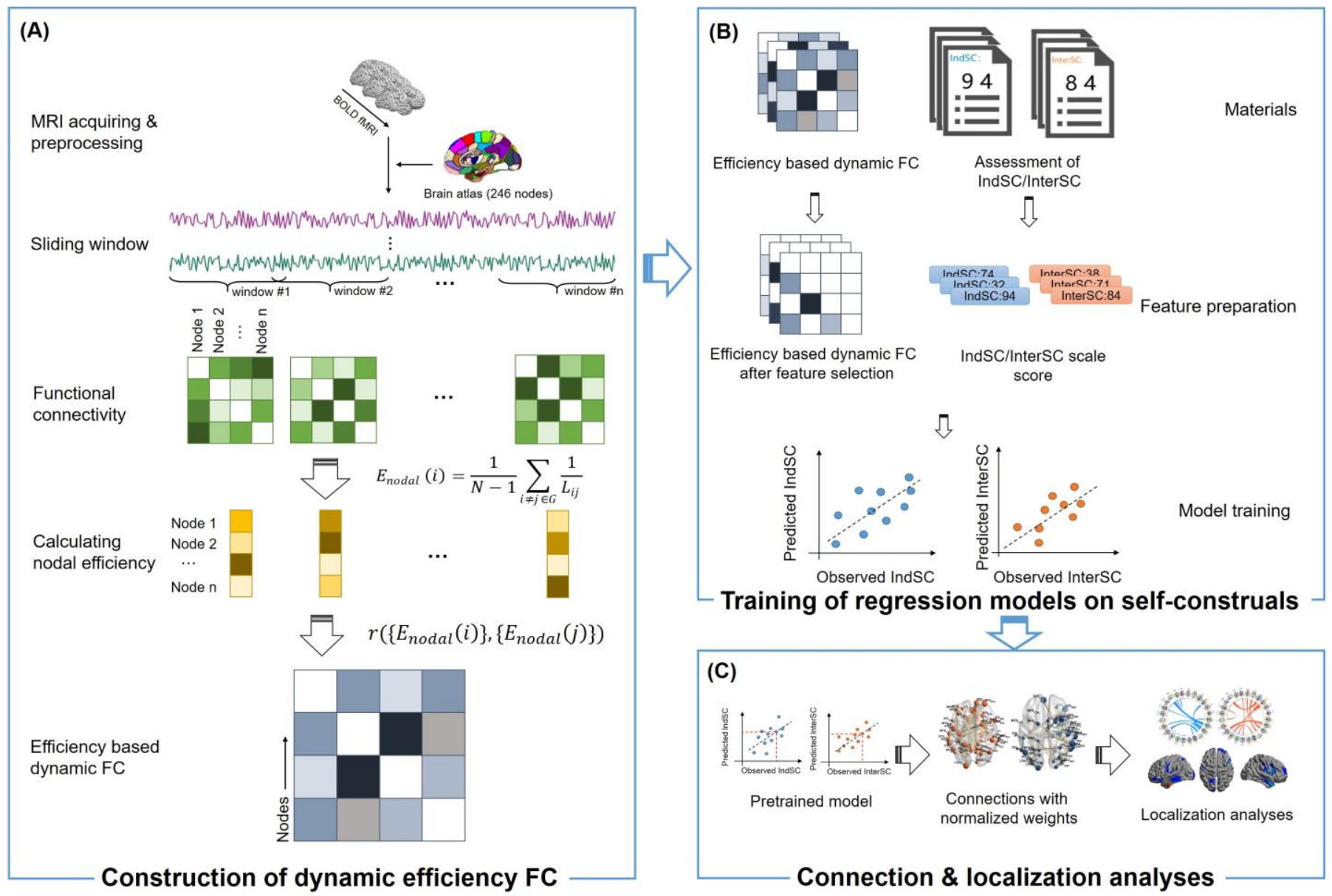

Fig. 1. A schematic illustration of the proposed analysis approach. (A) The preprocessed resting-state BOLD fMRI time series are firstly extracted and parceled with Brainnetome Atlas. Dynamic functional connectivity is then constructed using the sliding window method, with the window length of $22 \mathrm{TRs}(50.6 \mathrm{~s})$, and the sliding step size of 1 TR $(2.3 \mathrm{~s})$. The Nodal efficiency of each node is calculated for every window. For each participant, the correlation coefficient between each pair of nodal efficiency time series is calculated, which forms a $246 \times 246$ correlation matrix, termed as efficiency-based dynamic FC in this study. (B) Utilizing efficiency-based dynamic FC obtained from (A), regression models are used to assess the relationship between dynamic brain networks and the assessment of self-construal (i.e. independent self-construal and interdependent self-construal). (C) After using nested 10-fold-cross-validation to demonstrate the validity of the regression model, we make the model output the weights of each connection and then normalize these weights, thereby performing analysis and mapping the relationship between brain dynamic activity and the score of self-construal.

TABLE I

PARTICIPANT DEMOGRAPHIC AND CLINICAL INFORMATION (N=323)

\begin{tabular}{llll}
\hline \hline \multicolumn{1}{c}{ Item } & \multicolumn{1}{c}{ Description } & $\begin{array}{c}\text { Correlation with } \\
\text { the IndSC }\end{array}$ & $\begin{array}{c}\text { Correlation with } \\
\text { the InterSC }\end{array}$ \\
\hline Age & $22.75 \pm 2.97(\mathrm{SD})$ & $-0.02(p=0.71)$ & $0.03(p=0.60)$ \\
Gender & $159(\mathrm{M}): 164(\mathrm{~F})$ & $-0.08(p=0.15)$ & $-0.01(p=0.80)$ \\
Hand use & $315(\mathrm{R}): 8(\mathrm{~L})$ & $0.03(p=0.61)$ & $-0.01(p=0.79)$ \\
IndSC & $72.54 \pm 9.75(\mathrm{SD})$ & $1.00(p=0.0)$ & $0.25(p=5.77 \mathrm{E}-6)$ \\
InterSC & $76.39 \pm 9.32(\mathrm{SD})$ & $0.25(p=5.77 \mathrm{e}-6)$ & $1.00(p=0.0)$ \\
\hline \hline
\end{tabular}

this study, the alpha coefficient for the IndSC and InterSC subscales were set to 0.75 according to our previous research [40, 41]. IndSC and InterSC scores of all participants cover a relatively complete and continuous range (Table 1).

\section{Image Data Acquisition}

Functional images were collected on a 3T Philips Achieva MRI scanner (Philips Healthcare, Best, The Netherlands) with a 32-channel head coil. Head motion was controlled by the tight but comfortable foam padding.

Resting-state blood-oxygenation-level Dependent (BOLD) signals were collected using a gradient-echo-planar imaging sequence with the following parameters: 37 axial slices; repetition time $(\mathrm{TR})=2300 \mathrm{~ms}$; echo time $(\mathrm{TE})=35 \mathrm{~ms}$; flip angle $(\mathrm{FA})=90^{\circ} ;$ slice thickness $=2.5 \mathrm{~mm}$; gap $=1.0 \mathrm{~mm}$; matrix $=96 \times 96$; field of view $=240 \times 240 \mathrm{~mm}^{2}$. During the scan, the participants were guided to lie inactive and motionless in the scanner, with eyes closed but staying awake, keeping relaxed without thinking specific things in deliberate. The whole scan lasted for 508.3 seconds.

The high-resolution T1-weighted SPGR structural images in coronal view were acquired with the slice thickness of $1 \mathrm{~mm}$ without gap. Other sequence parameters were: TR/TE $=8.1 / 3.7$ $\mathrm{ms}, \mathrm{FOV}=240 \times 240 \mathrm{~mm}^{2}$, matrix $=240 \times 240 \times 160$.

\section{Data Preprocessing}

Functional images were preprocessed using the GRETNA [42] and SPM12 toolkits (www.fil.ion.ucl.ac.uk/spm). The preprocessing of rs-fMRI data included discarding the first 10 volumes to ensure magnetization equilibrium, slice timing correction with the first slice, and head-motion correction. The $0.01-0.10 \mathrm{~Hz}$ frequency band-pass were used. The nuisance signal regression (24-parameter head motion profiles, global signal, CSF signal, and WM signal) was performed. In order to do the group analysis, the first volume of the fMRI time series that were not discarded was coregistered to the same 
participant's T1-weighted images, and then normalized to the Montreal Neurological Institute (MNI) template space.

\section{E. Construction of Dynamic Networks}

\section{1) Node Definition}

The parcellation of brain is defined by a connectional architecture brain atlas named Brainnetome Atlas [43] with 246 nodes. This method takes the advantage of considering functionally important connectivity information on both anatomical and functional connections via spectral clustering that based on the similarity among connectivities.

2) Static functional connectivity

GRETNA software was used to construct the whole-brain networks. As a comparison with our proposed method, the static functional connectivity analysis is carried out by the following process: the mean signal of each region of interest (ROI) was obtained by averaging the BOLD time series over all voxels within that region. The edges of the static functional brain networks were computed by Pearson correlation coefficient (r). Eventually, a $246 \times 246$ matrix was derived for each participant, and further network analysis was conducted on the basis of such matrix.

\section{3) Dynamic functional connectivity}

The dynamic functional connectivity was calculated by using a sliding window approach. The sliding-window approach was used to explore the time-varying changes of functional connectivity within the 246 nodes during functional MRI scans. The resting state time series data were segmented into a 22-TR window with the size of $50.6 \mathrm{~s}$ ( $2.3 \mathrm{~s}$ per TR). The window was slided step-wise by $1 \mathrm{TR}$ along the 221-TR length scan (i.e. $508.3 \mathrm{~s}$ ), resulting in 200 consecutive windows across the entire scan. The reason of choosing 22 TRs as the segmented window length is that it has been reported to provide a good trade-off between the ability to resolve dynamics of functional connectivity and the quality of the correlation matrix estimation [28, 29].

\section{F. Calculating Efficiency-Based Dynamic FC}

As a topological property in network analysis, network efficiency is considered to measure the global efficiency of the parallel information transfer in the network. The global network efficiency describes the whole-brain network [44-46].Similarly, to determine the nodal (regional) characteristics of the networks, we computed the nodal efficiency, $E_{\text {nodal }}(i)$ [47]:

$$
E_{\text {nodal }}(i)=\frac{1}{N-1} \sum_{i \neq j \in G} \frac{1}{L_{i j}}
$$

where $L_{i j}$ is the shortest path length between nodes $i$ and $j$ in the entire brain network $G . E_{\text {nodal }}(i)$ measures the average shortest path length between a given node $i$ and all of the other nodes in the network. Note that the length was defined according to the distance between two coordinates of nodes from Brainnetome Atlas.

In this study, for each sliding window of a participant, the efficiency of each brain region is computed. It results in a 246 $\times 1$ nodal efficiency vector. By joining nodal efficiency vectors from 200 windows, a matrix with a shape of $246 \times 200$ is then formed. In other words, an array of 200 numbers was formed by its variation in the sliding windows for each node. By calculating the Pearson's correlation coefficient $r$ between each pair of the array, the efficiency-based functional correlation matrix, termed efficiency-based dynamic FC, is obtained for one participant (Fig 1A), and the final dimension of efficiencybased dynamic FC is $246 \times 246$. With this approach, the efficiency-based functional correlation matrix describes the correlation of dynamic changes in nodal efficiency between two nodes.

\section{G. Mapping the Relationship between Efficiency-Based Dynamic FC with Self-construals by Using Regression Model}

To further investigate the effectiveness of efficiency-based dynamic FC in recognizing self-construals (Fig 1B), we performed regression model analysis to learn the relationship between the dynamic FC matrix and scores of IndSC/InterSC. Due to the large dimension of the efficiency-based dynamic FC matrix, we established a feature selection criterion. That is, we only kept the dimensions whose $\mathrm{p}$-value of correlation with the SCS scores were less than 0.05 in the matrix (Fig 2A) for further analysis. It should be noted that the feature selection is only applied on the training set for each training iteration to avoid potential overfitting problem.

Thus, we utilized XGBoost as the regression model in the following experiment. To examine whether efficiency-based dynamic FC can be used to correctly extract meaningful connections for identifying individuals' IndSC/InterSC, we utilized nested 10-fold cross-validation to evaluate the performance of the regression model, thereby avoiding potential overfitting and deriving the optimal parameters [48]. For each time, one in ten individuals' IndSC/InterSC as well as their efficiency-based dynamic FC data were kept as validation data, while the rest were used as training data of the regression model. The regression model needs to learn the relationship between the efficiency-based dynamic FC and the corresponding self-construals in the training set and make a prediction on the efficiency-based dynamic FC matrix in the test set. The difference between the actual score of selfconstrual and the prediction was recorded as regressing error.

To measure the performance of the regression model, we used mean absolute error (MAE) and mean square error (MSE) to calculate the quantitative error between the predicted and the real IndSC/InterSC scores. In addition, the Spearman correlation coefficient was used to measure the correlation between the predicted and the actual scores. In addition, a permutation test was also applied to investigate whether the obtained metrics were significantly better than expected by chance. In the experiment, we permuted IndSC/InterSC scores across all participants with 1,000 times under same hyperparameters condition, and each time re-applied the above nested cross-validation procedure. This resulted in a distribution of correlation (r) and MSE values reflecting the null hypothesis that the model did not exceed chance. The number of times that when the permuted value was greater than (or with respect to MAE and MSE values, less than) the true value was then divided by 1,000 to provide an estimated $\mathrm{p}$-value for the correlation coefficient, observed MSE and MAE [49]. 


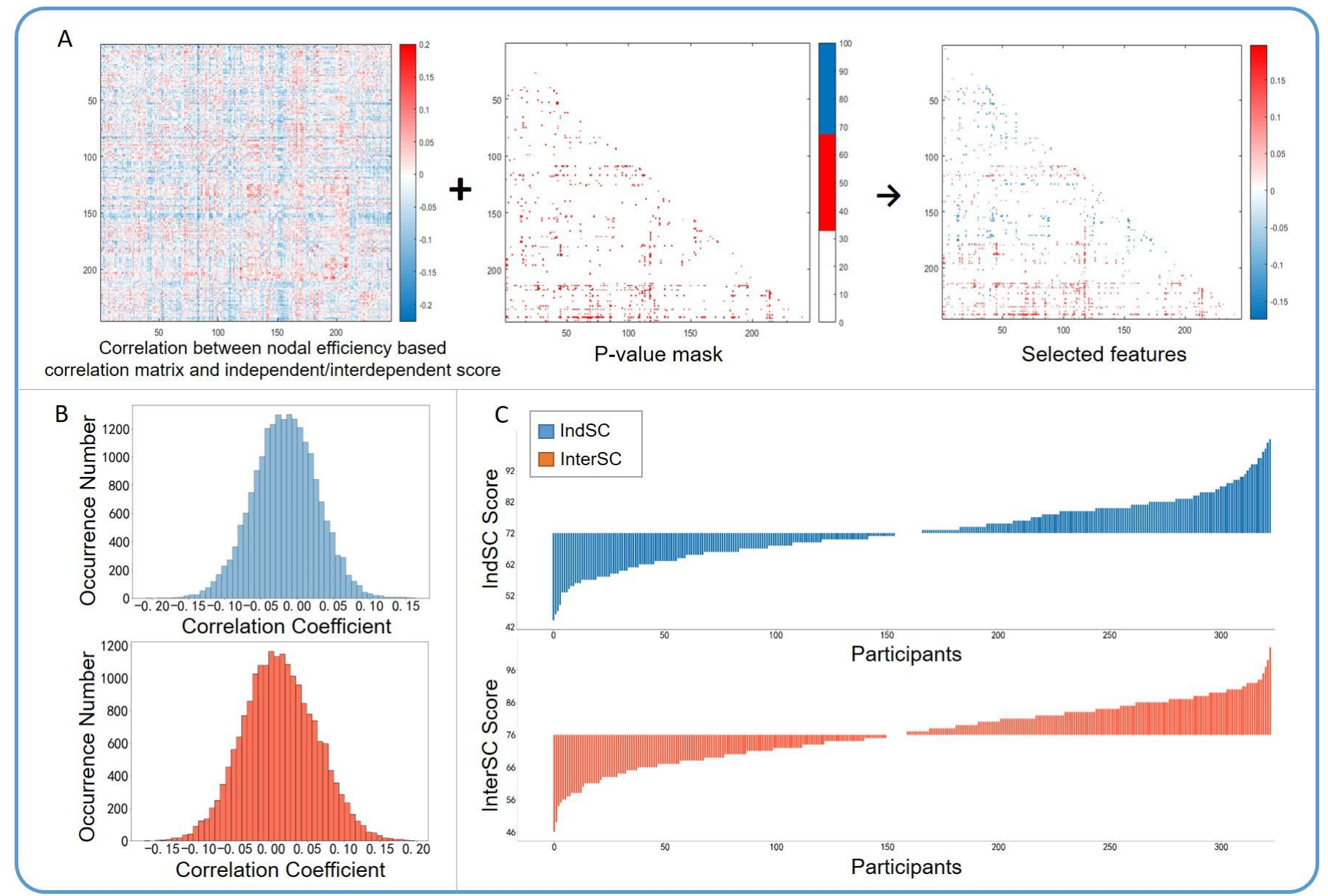

Fig. 2. Feature selection scheme and the scores of independent/interdependent self-construal. (A) Pearson correlations between nodal efficiency of each pair of brain nodes for each participant are first calculated to construct individual efficiency-based dynamic FC. Pearson's coefficient (r) between each dimension of efficiency-based dynamic FC of all participants are then computed and their correlation scores with statistical significance (p-value $<0.05)$ are kept as features for the regression model. (B) Distribution of Pearson coefficient values for connection in our proposed dynamic functional connectivity and independent/interdependent self-construals. (C) Distribution of independent/interdependent self-construal scores across participants.

We also compared the predicting performance across the proposed efficiency based dynamic FC, static FC and nodal efficiency. Specifically, the static FC refers to the standard static functional connectome based on Pearson correlation between time courses of each brain region, and it is a $246 \times 246$ dimensional matrix for each participant. The nodal efficiency is calculated on the entire time course of each brain region.

\section{H. Regression model weighted connection analysis}

After selecting the parameters with the best performance, we further examined the important connection by referring the importance it contributed to the regression model. Specifically, we presented the important brain nodes and connections (i.e. nodes and edges in the efficiency-based dynamic FC) selected by the XGBoost model with the best regression performance (i.e. smallest MAE/MSE and largest $r$ ). In particular, the importance of each connection is selected according to the model alongside with nested 10 -fold cross-validation, then each importance is normalized and averaged as final connection coefficient.

Furthermore, based on the differences in left and right brain functions of self-construction reported in previous research $[4$, 20], we have calculated number and proportion of the two nodes connected by one connection which are completely located in the left hemisphere, right hemispheres and connections across the both hemisphere in the top $\mathrm{N}$ important connections selected by the regression model. In particular, BrainNet Viewer [50] was used to map nodes and connections to exhibit model selected significant connections and coefficients based brain maps.

\section{RESULTS}

\section{A. Demographic Characteristics}

As described in the previous section, the SCS scores of all participants were consistently and evenly distributed (Fig 2C) with respect to the degree of independent/interdependent orientations. In addition, the scores of independence and interdependence were not significantly correlated with participants' age, gender, or hand use (Table 1).

\section{B. Validating the Performance of Regression Models Using Nested Cross-Validation}

In the 10-fold cross-validation, the MAE, MSE and correlation coefficient between the predicted and the true SCS scores were obtained to evaluate the performance. Shown in Table 2, the XGBoost regression model reached a comparative 

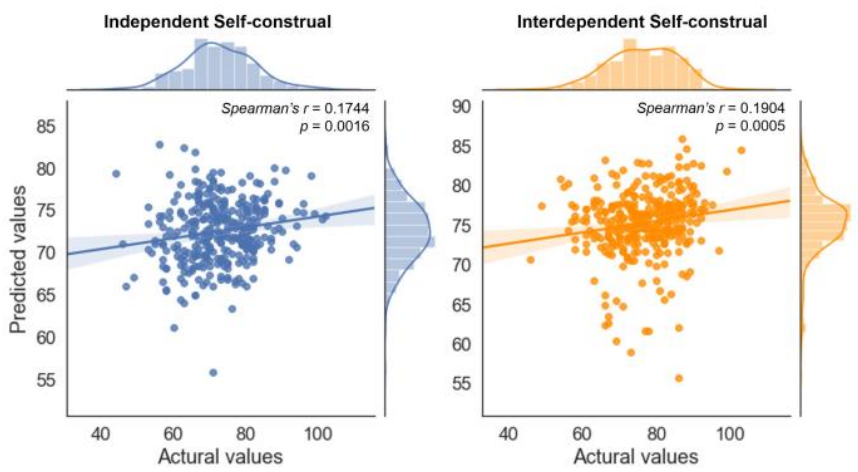

Fig. 3. Correlations between actual and predicted scores of independent and interdependent self-construals with XGBoost regression.

quantitative error (MAE and MSE) for predicting both IndSC (MAE $=7.83$, MSE $=96.05, p$-value of permutation test $<0.001$ ) and InterSC scores (MAE=7.75, MSE=93.43, $p$-value of permutation test $<0.001$ ). The Spearman correlation coefficient between the predicted and the actual values are 0.17 and 0.19 , respectively (Fig 3). All results passed Bonferroni corrections for the multiple comparisons. This indicate that the dynamic correlation between the node efficiency we extracted can effectively help to estimate self-construals.

Furthermore, to demonstrate the superiority of efficiencybased dynamic FC in the presented method over static FC as well as nodal efficiency itself, we performed an experiment by using static rsFC or nodal efficiency on time course to predict individual self-construals under same experiment protocol. Steiger test is performed to compare the significance of dependent correlation coefficients $[51,52]$. As a result, the zscores of Steiger test between the proposed FC and static FC are 1.036 for IndSC and 2.240 for InterSC. The z-scores of Steiger test between the proposed FC and nodal efficiency are 1.380 for IndSC and 3.987 for InterSC, respectively. This result showed that the proposed InterSC identification has a statistically significant improvement compared with other existing features. The comparison also showed that the proposed method reduced the MAE result by 1.4 in IndSC regression and 0.4 in InterSC regression, respectively (Table 2). Depending on the model's interpretability [53], we use the model outputted importance for subsequent analysis.

\section{Localization of Model Selected Connections and Regions}

An exploratory correlation analysis was conducted across all participants to examine the relevance of efficiency-based functional correlation matrix to IndSC and InterSC. To be specific, we used the XGBoost model which had the optimal performance to output the importance of each connection. We selected the top $\mathrm{N}(\mathrm{N}=10,20,30$, and 40) connections according to the largest $\mathrm{N}$ average normalized coefficients of the XGBoost model, and then categorized these connections into three types, i.e., left-hemisphere, right-hemisphere and cross-hemisphere connections, according to whether two nodes of a certain connection are both located in the left/right hemisphere or they are located in two different hemispheres. The difference in hemispherical polarities of the connections in IndSC and InterSC regression models are confirmed by chi-square test. As a result, IndSC exhibits an obvious right-brain dominance, while InterSC, on the contrary, exhibits an obvious lefthemisphere dominance. For instance, in the 20 most important connections, more than a half $(12,60 \%)$ left or righthemisphere connections was observed for IndSC. Whereas for InterSC, left-hemisphere connections played a more significant role $(12,60 \%)$ (Fig 4A).

We also investigated the important regions that effects the regression of the two self-construals. Selected by the coefficients of regions outputted by the regression model, the top 10 important connections are listed in Table 3. For each connection, the weighted coefficient is assigned equally to the node it linked to. Then by overlaying all connections' coefficients, the coefficients map is obtained, as presented in Fig 5. Observed important regions, including Temporal Lobe $\left(\mathrm{PhG}_{\mathrm{L} 66}, \mathrm{ITG}_{\mathrm{R} 71}, \mathrm{ITG}_{\mathrm{L} 77}, \mathrm{STG}_{\mathrm{L} 64}, \mathrm{STG}_{\mathrm{R} 62}\right)$, Frontal Lobe $\left(\mathrm{PrG}_{\mathrm{R} 61}, \mathrm{MFG}_{\mathrm{R} 76}, \mathrm{OrG}_{\mathrm{R} 63}\right)$, Occipital Lobe $\left(\mathrm{LOcC}_{\mathrm{R} 41}, \mathrm{LOcC}_{\mathrm{L} 21}\right.$, $\left.\mathrm{MVOcC}_{\mathrm{R} 55}, \mathrm{LOcC}_{\mathrm{L} 41}, \mathrm{LOcC}_{\mathrm{R} 43}\right)$, Parietal Lobe $\left(\mathrm{PoG}_{\mathrm{R} 41}\right.$, $\mathrm{PoG}_{\mathrm{R} 43}$ ), Insular Lobe $\left(\mathrm{INS}_{\mathrm{R} 64}\right)$, and part of Limbic Lobe $\left(\mathrm{CG}_{\mathrm{R} 75}\right)$, made the major contribution to identify the IndSC. On the other hand, the regions from Frontal Lobe $\left(\mathrm{OrG}_{\mathrm{L} 62}, \mathrm{MFG}_{\mathrm{L} 77}\right.$, $\left.\mathrm{MFG}_{\mathrm{L} 71}, \mathrm{OrG}_{\mathrm{L} 64}\right)$, Temporal Lobe $\left(\mathrm{PhG}_{\mathrm{L} 63}, \mathrm{ITG}_{\mathrm{L} 75}, \mathrm{MTG}_{\mathrm{L} 43}\right.$, $\mathrm{PhG}_{\mathrm{L} 65}$ ), Subcortical Nuclei (Tha ${ }_{\mathrm{L} 83}$, Amyg $_{\mathrm{R} 21}$, Tha ${ }_{\mathrm{L} 87}, \mathrm{BG}_{\mathrm{R} 65}$, Tha ${ }_{\text {R4 }}$ ), and Limbic Lobe (CGL73, CGL75) have made the major contribution to the process of identifying InterSC (further anatomical and modified Cyto-architectonic descriptions can be accessed at http://atlas.brainnetome.org). From these results, we observed that Occipital Lobe, Parahippocampal Gyrus, Middle Frontal Gyrus, Orbital Gyrus in the Frontal Lobe, Middle Temporal Gyrus, Inferior Temporal Gyrus in the Temporal Lobe, Subcortical Nuclei, and part of the limbic system are involved in the processing of both IndSC and InterSC. Although there are many similarities in the lobe level, the regions and sub-regions that influence the independent and interdependent self still have large differences (Table 3, Fig 4B). While in the IndSC and InterSC regression models, there are no concurrent connections among the top 40 important connections with the largest coefficients.

Although these important areas are located in different lobes, Frontal Lobe and Temporal Lobe still make a major contribution to identifying the self-construals. In the IndSC regression analysis, the number of connections in which Temporal Lobe is involved achieves 6, and the number of connections in which Frontal Lobe is involved achieves 3 in the top 10 connections. Similar proportions can also be observed as the value of top $\mathrm{N}$ is continuously expanded. In the analysis of identifying InterSC, there are 4 connections in which Temporal Lobe is involved and 5 connections in which Frontal Lobe is involved, in the top 10 connections. In addition, the connections between Temporal Lobe and Frontal Lobe also showed their importance to affecting the regression. There are 6 connections connecting Temporal Lobe in the task of identifying IndSC and 4 connection located in Frontal Lobe. The above results also reflect that both self-construals are similar many cognitive traits that majorly processed in Frontal and Temporal lobe, InterSC processing relies more on the collaborative processing of multiple brain lobes, while IndSC relies more on the connection 


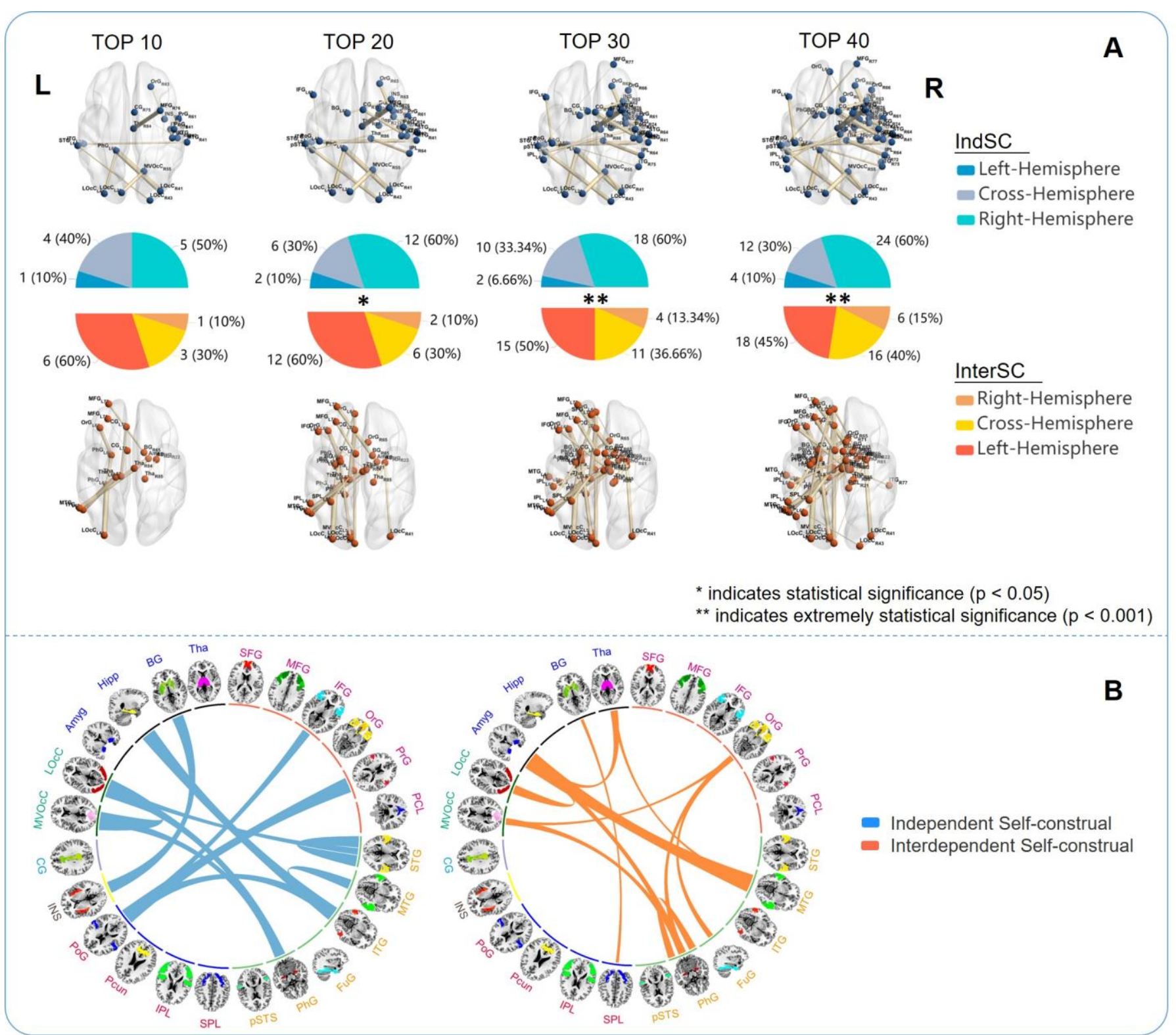

Fig. 4. Model outputted important nodes and correlations of nodal efficiency (features) between nodes. (A) Top $N$ ( $N=10,20,30,40)$ correlations of nodal efficiency which were selected by the coefficient of the regression model. (B) From specific brain node scaling to more macroscopic networks, correlations of nodal efficiency represent a broader range of cross-network attributes in both the orientation of independence and interdependence $(\mathrm{N}=20)$.

TABLE II

ERRORS AND CORRELATIONS BASED ON THE XGBOOST REGRESSION MODEL FOR IDENTIFYING INDEPENDENT SELF-CONSTRUAL (INDSC) AND INTERDEPENDENT SELF-CONSTRUALS (INTERSC) WITH DIFFERENT FC BY USING NESTED 10-FOLD-CROSS-VALIDATION

\begin{tabular}{|c|c|c|c|c|c|c|}
\hline Self-construals & Feature & Spearman's r & MAE & MSE & $\begin{array}{c}p \text {-value of } \\
\text { Spearman's Correlation }\end{array}$ & $\begin{array}{c}p \text {-value of } \\
\text { permutation test }\end{array}$ \\
\hline \multirow{3}{*}{ IndSC } & Efficiency-based dynamic FC & 0.17 & 7.83 & 96.05 & 0.0016 & $<0.001$ \\
\hline & Static FC & 0.09 & 9.23 & 140.24 & 0.0995 & 0.032 \\
\hline & Nodal efficiency & 0.07 & 7.98 & 104.23 & 0.1801 & 0.070 \\
\hline \multirow{3}{*}{ InterSC } & Efficiency-based dynamic FC & 0.19 & 7.75 & 93.43 & 0.0005 & $<0.001$ \\
\hline & Static FC & 0.01 & 8.15 & 106.43 & 0.8221 & 0.246 \\
\hline & Nodal efficiency & -0.12 & 8.59 & 110.80 & 0.1293 & 0.639 \\
\hline
\end{tabular}

between frontal and temporal related regions

\section{DISCUSSION}

Self-construal is one of the complex cultural-related concepts that have drawn considerable attention from psychologists and neuroscientists in the past two decades $[54,55]$. Using machine learning approaches, we incorporated nodal efficiency based dynamic correlations into rs-fMRI data to capture higher-order cognitive attributes in the whole-brain functional connectivity. We then derived the connectome patterns associated with the orientations of independence and interdependence through the efficiency-based dynamic FC.

Specifically, we obtained the time-varying behavior of each nodal efficiency. It should be noted that efficiency is a graphic 
TABLE III

THE TOP 10 IMPORTANT CONNECTIONS CONTRIBUTED TO IDENTIFYING THE INDEPENDENT SELF-CONSTRUAL (INDSC) AND INTERDEPENDENT SELF-CONSTRUAL (INTERSC), WHICH WAS SELECTED BY WEIGHTED IMPORTANCE OF THE REGRESSION MODEL.

\begin{tabular}{|c|c|c|c|c|}
\hline $\begin{array}{c}\text { Self- } \\
\text { construals }\end{array}$ & No & Region (Lobe) & Region (Lobe) & $\begin{array}{c}\text { Weighted } \\
\text { Coefficient }\end{array}$ \\
\hline \multirow{10}{*}{ IndSC } & 1 & LOcC $_{\mathrm{R} 41}$ (Occipital Lobe) & PhGL66 (Temporal Lobe) & 0.37 \\
\hline & 2 & PoGR41 (Parietal Lobe) & PrGR61 (Frontal Lobe) & 0.34 \\
\hline & 3 & ThaR84 (Subcortical Nuclei) & MFGR76 (Frontal Lobe) & 0.34 \\
\hline & 4 & LOcC $_{\text {L21 }}$ (Occipital Lobe) & $\mathrm{MVOcC}_{\mathrm{R} 55}$ (Occipital Lobe) & 0.32 \\
\hline & 5 & PoG $_{\mathrm{R} 43}$ (Parietal Lobe) & ITGR71 (Temporal Lobe) & 0.29 \\
\hline & 6 & LOcC $_{\text {L41 }}$ (Occipital Lobe) & PhGl66 (Temporal Lobe) & 0.26 \\
\hline & 7 & ITG $_{\text {L77 }}$ (Temporal Lobe) & $\mathrm{MTG}_{\mathrm{R} 41}$ (Temporal Lobe) & 0.26 \\
\hline & 8 & LOcC $_{\mathrm{R} 43}$ (Occipital Lobe) & STGL64 (Temporal Lobe) & 0.24 \\
\hline & 9 & $\mathrm{INS}_{\mathrm{R} 64}($ Insular Lobe) & OrGR63 (Frontal Lobe) & 0.24 \\
\hline & 10 & CGR75 (Limbic Lobe) & STG $_{\mathrm{R} 62}$ (Temporal Lobe) & 0.23 \\
\hline \multirow{10}{*}{ InterSC } & 1 & LOcC $_{\text {L41 }}$ (Occipital Lobe) & PhGL63 (Temporal Lobe) & 0.60 \\
\hline & 2 & ThaL83 (Subcortical Nuclei) & ITGL75 (Temporal Lobe) & 0.52 \\
\hline & 3 & Amyg $_{R 21}$ (Subcortical Nuclei) & MTGL43 (Temporal Lobe) & 0.45 \\
\hline & 4 & PhGL65 (Temporal Lobe) & OrGL62 (Frontal Lobe) & 0.42 \\
\hline & 5 & PhGL65 (Temporal Lobe) & $\mathrm{PhG}_{\mathrm{L} 63}$ (Temporal Lobe) & 0.40 \\
\hline & 6 & ThaL87 (Subcortical Nuclei) & CGL73 (Limbic Lobe) & 0.39 \\
\hline & 7 & $\mathrm{BG}_{\mathrm{R} 65}$ (Subcortical Nuclei) & $\mathrm{MFG}_{\mathrm{L} 77}$ (Frontal Lobe) & 0.37 \\
\hline & 8 & Thar84 (Subcortical Nuclei) & MTGL43 (Temporal Lobe) & 0.36 \\
\hline & 9 & CGL75 (Limbic Lobe) & MFGL71 (Frontal Lobe) & 0.35 \\
\hline & 10 & $\mathrm{MVOcC}_{\mathrm{L} 53}$ (Occipital Lobe) & OrG 64 (Frontal Lobe) & 0.34 \\
\hline
\end{tabular}

Note. The full name and anatomical description of each region can be found at http://atlas.brainnetome.org/.
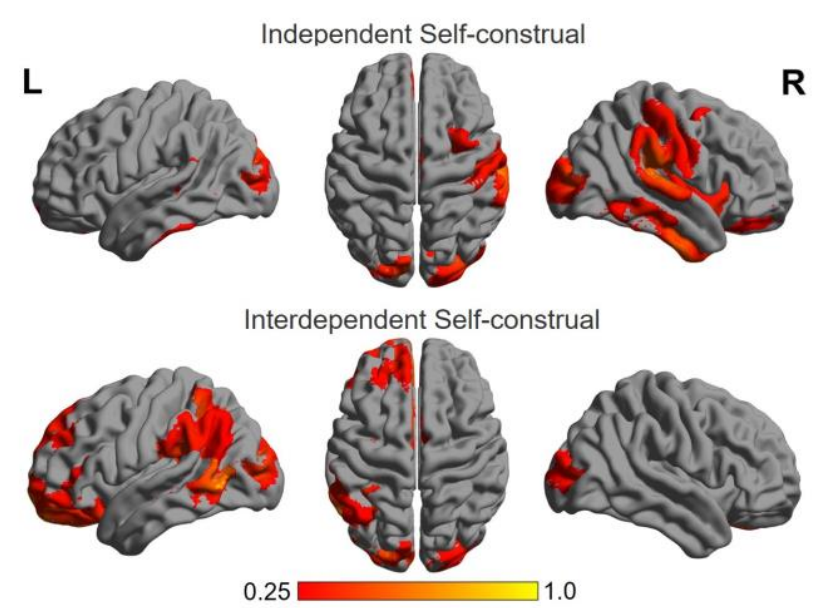

Fig. 5. Coefficient map of independent self-construal and interdependent selfconstrual regressors. Coefficients are determined by the regression model and normalized.

property that quantifies the degree of easiness of information exchange over the graph. This measurement provides information on the communication efficiency of a graph as a whole, with higher values indicating more efficient information transferring through the whole brain. A variety of graphic properties located in dynamic $\mathrm{FC}$ has yielded novel insights into brain function and dysfunction [37]. Thus, the functional synergy of brain regions in the rs-fMRI could be extracted using our proposed approach. The experimental results suggested that the brain is organized in a highly efficient small-world topology, combining a high level of segregation (local or nodal efficiency) with a high level of global integration (global efficiency) [47]. In other words, the reason for using nodal efficiency is that it represents a graphic property that quantifies the degree of ease of information exchange over a graph. This measurement provides information on the communication efficiency of the graph as a whole, with higher values indicating more efficient information transfer through the whole brain. The relationship between the psychological traits and combination of different graph properties and their format of different graph properties (such as causality, correlation, mean value, and method) remains unclear, which invites future investigations.

Going beyond previous studies that investigated the relationship between brain localization and the process of the self-construal, we demonstrated that the brain connectivity across different regions and lobes contributed to individual variations in trait IndSC and InterSC. Previous research reported that activity in the frontal cortex, including the medial prefrontal cortex (mPFC), was enhanced during general trait and contextual trait judgments. These regions are thought to associate with self-knowledge and self-construals [15, 19, 5659]. Our results showed that functional connectivity across the mPFC, the parahippocampal gyrus, cingulate gyrus, insular gyrus, and the middle frontal gyrus linked to independence and interdependence, consistent with the previous studies [10, 60, 61]. We did not observe the connectivity through the precuneus and the inferior frontal gyrus reported by $\mathrm{Li}$ et al [22]. This discrepancy may be due to the fact that the present study used the dynamic correlation between the efficiency of different nodes, rather than the voxel-based brain activities used in the previous study. As suggested by electroencephalography (EEG) studies, it is sensible to use dynamic approaches to test complex psychological processes and concepts (e.g., self-construal), since they are associated with multiple brain regions and lobes, such as the frontal lobe, temporal lobe, and limbic systems [17, 62-66].

Mapping the process mechanism of social or psychological property in the human brain is a long-term research focus. Our results suggest that dynamic functional connectomes are 
sensitive to characterize trait independence and trait interdependence, and that neurobiological-based dynamic fMRI data and machine learning frameworks provide pivotal ingredients toward cultural neuroscience. To some extent, this connection based approach performs similarly to the recently focused significance of white matter in social and psychological behavior analysis, especially in social cognition [24]. Currently, the barrage of advances reveals that neurobiological-based brain dynamic activity has enormous potential to act as steady and efficient biomarkers in a number of neurological and neuropsychiatric disorders [67].

A limitation to the current study is that the whole-brain rsfMRI was used to mine the relationship between brain functional connectivity and self-construals through efficiencybased dynamic FC. Although this method effectively alleviates the traditional problems that there are few conspicuous features for identifying self-construals with static functional connectivity, the physiological and cognitive basis of the changes in the nodal efficiency of each two nodes is not clear. Another limitation is that current feature selection is based on statistical significance between connection features and psychological scores. Future studies may utilize graph clustering approaches to select representative clusters, thereby avoiding redundant features added to the regression model. Furthermore, the generalizability of the current results may be limited by the sample. Our results also require external validation to validate the degree and scale of the generalization of the proposed method [68]. Previous research has shown the asymmetry of independence and independence scores; hence, we took them as two independent scores in the present study. However, it is worth noting that there is an existing argument is self-construal unidimensional or multi-dimensionality selfconstrual $[12,40]$. These questions invite future investigations.

\section{CONCLUSION}

In this paper, we proposed a machine learning-based analysis framework for self-construals by using whole-brain efficiencybased dynamic FC. We demonstrated the feasibility of the regression models which identify individual variations in selfconstruals via their brain dynamic connectivity. We showed the commonality and distinctions of the dynamic functional connectivity that trait independence and trait interdependence. These findings provide the pivotal ingredients to the biological essence of culturally related variables in the brain.

\section{ACKNOWLEDGEMENTS}

We thank all our participants who participated in this research. This research was partly supported by the National Natural Science Foundation of China (62071049, 61801026), Leverhulme Trust (RPG-2019-010), and Beihang University \& Capital Medical University Advanced Innovation Center for Big Data-Based Precision Medicine Plan (BHME-201907).

\section{REFERENCES}

[1] H. R. Markus, and S. Kitayama, "Culture and the Self - Implications for Cognition, Emotion, and Motivation," Psychological Review, vol. 98, no. 2, pp. 224-253, Apr, 1991.
[2] S. E. Cross, and L. Madson, "Models of the self: Self-construals and gender," Psychological Bulletin, vol. 122, no. 1, pp. 5-37, Jul, 1997.

[3] D. Oyserman, H. M. Coon, and M. Kemmelmeier, "Rethinking individualism and collectivism: Evaluation of theoretical assumptions and meta-analyses," Psychological Bulletin, vol. 128, no. 1, pp. 3-72, Jan, 2002.

[4] S. E. Cross, E. E. Hardin, and B. Gercek-Swing, "The What, How, Why, and Where of Self-Construal," Personality and Social Psychology Review, vol. 15, no. 2, pp. 142-179, May, 2011.

[5] M. Adriani, A. Bellmann, R. Meuli, E. Fornari, R. Frischknecht, C. Bindschaedler, F. Rivier, J. P. Thiran, P. Maeder, and S. Clarke, "Unilateral hemispheric lesions disrupt parallel processing within the contralateral intact hemisphere: an auditory fMRI study," Neuroimage, vol. 20, pp. S66S74, Nov, 2003.

[6] H. Hitokoto, J. Glazer, and S. Kitayama, "Cultural shaping of neural responses: Feedback-related potentials vary with self-construal and face priming," Psychophysiology, vol. 53, no. 1, pp. 52-63, Jan, 2016.

[7] U. Hess, C. Blaison, and K. Kafetsios, "Judging Facial Emotion Expressions in Context: The Influence of Culture and Self-Construal Orientation," Journal of Nonverbal Behavior, vol. 40, no. 1, pp. 55-64, Mar, 2016.

[8] K. Sugimura, R. Nakama, S. Mizokami, K. Hatano, M. Tsuzuki, and S. J. Schwartz, "Working together or separately? The role of identity and cultural self-construal in well-being among Japanese youth," Asian Journal of Social Psychology, vol. 19, no. 4, pp. 362-373, Oct, 2016.

[9] X. R. Zhu, H. Y. Wu, S. Y. Yang, and R. L. Gu, "The influence of selfconstrual type on outcome evaluation: Evidence from event-related potentials," International Journal of Psychophysiology, vol. 112, pp. 6469, Feb, 2017.

[10] B. J. Liddell, K. L. Felmingham, P. Das, T. J. Whitford, G. S. Malhi, E. Battaglini, and R. A. Bryant, "Self-construal differences in neural responses to negative social cues," Biological Psychology, vol. 129, pp. 6272, Oct, 2017.

[11] J. Sui, and G. W. Humphreys, "The Integrative Self: How Self-Reference Integrates Perception and Memory," Trends in Cognitive Sciences, vol. 19, no. 12 , pp. 719-728, Dec, 2015.

[12] J. Sui, and X. Gu, "Self as Object: Emerging Trends in Self Research," Trends in neurosciences, vol. 40, no. 11, pp. 643-653, 2017/11//, 2017.

[13] S. H. Han, and Y. N. Ma, "A Culture-Behavior-Brain Loop Model of Human Development," Trends in Cognitive Sciences, vol. 19, no. 11, pp. 666-676, Nov, 2015.

[14] Y. Liu, B. Wu, C. Petti, X. Wu, and S. Han, "Self-construals moderate associations between trait creativity and social brain network," Neuropsychologia, vol. 111, pp. 284-291, 2018.

[15] J. G. Qin, S. Kimel, S. Kitayama, X. Y. Wang, X. D. Yang, and S. H. Han, "How choice modifies preference: Neural correlates of choice justification," Neuroimage, vol. 55, no. 1, pp. 240-246, Mar 1, 2011.

[16] M. E. W. Varnum, Z. H. Shi, A. T. Chen, J. Qiu, and S. H. Han, "When "Your" reward is the same as "My" reward: Self-construal priming shifts neural responses to own vs. friends' rewards," Neuroimage, vol. 87, pp. 164-169, Feb 15, 2014.

[17] C. B. Wang, Y. N. Ma, and S. H. Han, "Self-construal priming modulates pain perception: Event-related potential evidence," Cognitive Neuroscience, vol. 5, no. 1, pp. 3-9, Jan 2, 2014.

[18] J. Y. Chiao, T. Harada, H. Komeda, Z. Li, Y. Mano, D. Saito, T. B. Parrish, N. Sadato, and T. Iidaka, "Dynamic Cultural Influences on Neural Representations of the Self," Journal of Cognitive Neuroscience, vol. 22, no. 1, pp. 1-11, Jan, 2010.

[19] T. Harada, Z. Li, and J. Y. Chiao, "Differential dorsal and ventral medial prefrontal representations of the implicit self modulated by individualism and collectivism: An fMRI study," Social Neuroscience, vol. 5, no. 3, pp. 257-271, 2010.

[20] S. H. Ng, S. H. Han, L. H. Mao, and J. C. L. Lai, "Dynamic bicultural brains: fMRI study of their flexible neural representation of self and significant others in response to culture primes," Asian Journal of Social Psychology, vol. 13, no. 2, pp. 83-91, Jun, 2010.

[21] S. H. Han, G. Northoff, K. Vogeley, B. E. Wexler, S. Kitayama, and M. E. W. Varnum, "A Cultural Neuroscience Approach to the Biosocial Nature of the Human Brain," Annual Review of Psychology, Vol 64, vol. 64, pp. 335-359, 2013.

[22] L. M. W. Li, S. Luo, J. Ma, Y. Lin, L. Fan, S. Zhong, J. Yang, Y. Huang, L. Gu, L. Fan, Z. Dai, and X. Wu, "Functional connectivity pattern underlies individual differences in independent self-construal," Soc Cogn Affect Neurosci, vol. 13, no. 3, pp. 269-280, Mar 1, 2018. 
[23] J. Sui, R. Huster, Q. B. Yu, J. M. Segall, and V. D. Calhoun, "Functionstructure associations of the brain: Evidence from multimodal connectivity and covariance studies," Neuroimage, vol. 102, pp. 11-23, Nov 15, 2014.

[24] Y. Wang, and I. R. Olson, "The Original Social Network: White Matter and Social Cognition," Trends Cogn Sci, vol. 22, no. 6, pp. 504-516, Jun, 2018.

[25] O. Sporns, "Contributions and challenges for network models in cognitive neuroscience," Nature neuroscience, vol. 17, no. 5, pp. 652, 2014.

[26] M. Xia, and Y. He, "Functional connectomics from a "big data" perspective," NeuroImage, vol. 160, pp. 152-167, 2017

[27] G. J. Thompson, M. E. Magnuson, M. D. Merritt, H. Schwarb, W. J. Pan, A. McKinley, L. D. Tripp, E. H. Schumacher, and S. D. Keilholz, "ShortTime Windows of Correlation Between Large-Scale Functional Brain Networks Predict Vigilance Intraindividually and Interindividually," Human Brain Mapping, vol. 34, no. 12, pp. 3280-3298, Dec, 2013.

[28] E. A. Allen, E. Damaraju, S. M. Plis, E. B. Erhardt, T. Eichele, and V. D. Calhoun, "Tracking Whole-Brain Connectivity Dynamics in the Resting State," Cerebral Cortex, vol. 24, no. 3, pp. 663-676, Mar, 2014.

[29] J. Kim, M. Criaud, S. S. Cho, M. Diez-Cirarda, A. Mihaescu, S. Coakeley, C. Ghadery, M. Valli, M. F. Jacobs, S. Houle, and A. P. Strafella, "Abnormal intrinsic brain functional network dynamics in Parkinson's disease," Brain, vol. 140, pp. 2955-2967, Nov, 2017

[30] S. Dimitriadis, N. Laskaris, and S. Micheloyannis, "Transition dynamics of EEG-based network microstates during mental arithmetic and resting wakefulness reflects task-related modulations and developmental changes," Cognitive neurodynamics, vol. 9, no. 4, pp. 371-387, 2015.

[31] R. M. Hutchison, T. Womelsdorf, J. S. Gati, S. Everling, and R. S. Menon, "Resting-state networks show dynamic functional connectivity in awake humans and anesthetized macaques," Human Brain Mapping, vol. 34, no. 9, pp. 2154-2177, Sep, 2013.

[32]D. S. Bassett, and O. Sporns, "Network neuroscience," Nature Neuroscience, vol. 20, no. 3, pp. 353-364, Mar, 2017.

[33] O. Sporns, "Graph theory methods: applications in brain networks," Dialogues in Clinical Neuroscience, vol. 20, no. 2, pp. 111-120, Jun, 2018.

[34] G. Farnadi, G. Sitaraman, S. Sushmita, F. Celli, M. Kosinski, D. Stillwell, S. Davalos, M. F. Moens, and M. De Cock, "Computational personality recognition in social media," User Modeling and User-Adapted Interaction, vol. 26, no. 2-3, pp. 109-142, Jun, 2016.

[35] W. Bleidorn, and C. J. Hopwood, "Using Machine Learning to Advance Personality Assessment and Theory," pp. 1088868318772990, 2018.

[36] G. Varoquaux, "Cross-validation failure: Small sample sizes lead to large error bars," Neuroimage, vol. 180, pp. 68-77, Oct 15, 2018.

[37] X. Li, Y. Xiong, S. Liu, R. Zhou, Z. Hu, Y. Tong, L. He, Z. Niu, Y. Ma, and H. Guo, "Predicting the Post-therapy Severity Level (UPDRS-III) of Patients With Parkinson's Disease After Drug Therapy by Using the Dynamic Connectivity Efficiency of fMRI," Frontiers in neurology, vol. $10,2019$.

[38] D. Oyserman, S. Novin, N. Flinkenflögel, and L. Krabbendam, "Integrating culture-as-situated-cognition and neuroscience prediction models," Culture and Brain, vol. 2, no. 1, pp. 1-26, June 01, 2014.

[39] T. M. Singelis, "The Measurement of Independent and Interdependent Self-Construals," Personality and Social Psychology Bulletin, vol. 20, no. 5, pp. 580-591, Oct, 1994.

[40] F. Wang, K. Peng, M. Chechlacz, G. W. Humphreys, and J. Sui, "The Neural Basis of Independence Versus Interdependence Orientations: A Voxel-Based Morphometric Analysis of Brain Volume," Psychological Science, vol. 28, no. 4, pp. 519-529, 2017.

[41] J. Sui, and S. H. Han, "Self-construal priming modulates neural substrates of self-awareness," Psychological Science, vol. 18, no. 10, pp. 861-866, Oct, 2007.

[42] J. H. Wang, X. D. Wang, M. R. Xia, X. H. Liao, A. Evans, and Y. He, "GRETNA: a graph theoretical network analysis toolbox for imaging connectomics," Frontiers in Human Neuroscience, vol. 9, Jun 30, 2015.

[43] L. Z. Fan, H. Li, J. J. Zhuo, Y. Zhang, J. J. Wang, L. F. Chen, Z. Y. Yang, C. Y. Chu, S. M. Xie, A. R. Laird, P. T. Fox, S. B. Eickhoff, C. S. Yu, and T. Z. Jiang, "The Human Brainnetome Atlas: A New Brain Atlas Based on Connectional Architecture," Cerebral Cortex, vol. 26, no. 8, pp. 3508-3526, Aug, 2016.

[44]V. Latora, and M. Marchiori, "Efficient behavior of small-world networks," Physical Review Letters, vol. 87, no. 19, Nov 5, 2001.

[45] M. Rubinov, and O. Sporns, "Complex network measures of brain connectivity: Uses and interpretations," Neuroimage, vol. 52, no. 3, pp. 1059-1069, Sep, 2010.

[46] F. Bai, N. Shu, Y. G. Yuan, Y. M. Shi, H. Yu, D. Wu, J. H. Wang, M. R. Xia, Y. He, and Z. J. Zhang, "Topologically Convergent and Divergent
Structural Connectivity Patterns between Patients with Remitted Geriatric Depression and Amnestic Mild Cognitive Impairment," Journal of Neuroscience, vol. 32, no. 12, pp. 4307-4318, Mar 21, 2012.

[47] S. Achard, and E. T. Bullmore, "Efficiency and cost of economical brain functional networks," Plos Computational Biology, vol. 3, no. 2, pp. 174183, Feb, 2007

[48] Z. Cui, M. Su, L. Li, H. Shu, and G. Gong, "Individualized Prediction of Reading Comprehension Ability Using Gray Matter Volume," Cerebral Cortex, vol. 28, no. 5, pp. 1656-1672, 2018.

[49] C. Feng, J. Yuan, H. Geng, R. Gu, H. Zhou, X. Wu, and Y. Luo, "Individualized prediction of trait narcissism from whole-brain restingstate functional connectivity," Hum Brain Mapp, May 10, 2018

[50] M. R. Xia, J. H. Wang, and Y. He, "BrainNet Viewer: A Network Visualization Tool for Human Brain Connectomics," PloS One, vol. 8, no. 7, pp. e68910, Jul 4, 2013.

[51] M. Gao, C. H. Wong, H. Huang, R. Shao, R. Huang, C. C. Chan, and T. M. Lee, "Connectome-based models can predict processing speed in older adults," NeuroImage, vol. 223, pp. 117290, 2020.

[52] D. Scheinost, J. Dadashkarimi, E. S. Finn, C. G. Wambach, C. MacGillivray, A. L. Roule, T. A. Niendam, D. S. Pine, M. A. Brotman, and E. Leibenluft, "Functional connectivity during frustration: a preliminary study of predictive modeling of irritability in youth," Neuropsychopharmacology, pp. 1-7, 2021.

[53] K. Dadi, M. Rahim, A. Abraham, D. Chyzhyk, M. Milham, B. Thirion, G. Varoquaux, and A. D. N. Initia, "Benchmarking functional connectomebased predictive models for resting-state fMRI," Neuroimage, vol. 192, pp. 115-134, May 15, 2019.

[54] D. Oyserman, and S. W. S. Lee, "Does culture influence what and how we think? Effects of priming individualism and collectivism," Psychological Bulletin, vol. 134, no. 2, pp. 311-342, Mar, 2008.

[55] R. Gifford, and A. Nilsson, "Personal and social factors that influence proenvironmental concern and behaviour: A review," International Journal of Psychology, vol. 49, no. 3, pp. 141-157, Jun, 2014.

[56] K. N. Ochsner, J. S. Beer, E. R. Robertson, J. C. Cooper, J. D. Gabrieli, J. F. Kihsltrom, and M. J. N. D'Esposito, "The neural correlates of direct and reflected self-knowledge," vol. 28, no. 4, pp. 797-814, 2005.

[57] E. Magno, and K. J. P. S. Allan, "Self-reference during explicit memory retrieval: an event-related potential analysis," vol. 18 , no. 8, pp. $672-677$, 2007.

[58] J. Y. Chiao, T. Harada, H. Komeda, Z. Li, Y. Mano, D. Saito, T. B. Parrish, N. Sadato, and T. J. H. b. m. Iidaka, "Neural basis of individualistic and collectivistic views of self," vol. 30, no. 9, pp. 2813-2820, 2009.

[59] R. D. Ray, A. L. Shelton, N. G. Hollon, D. Matsumoto, C. B. Frankel, J. J. Gross, and J. D. E. Gabrieli, "Interdependent self-construal and neural representations of self and mother," Social Cognitive and Affective Neuroscience, vol. 5, no. 2-3, pp. 318-323, Jun, 2010.

[60] Y. Ma, C. Wang, B. Li, W. Zhang, Y. Rao, and S. Han, "Does selfconstrual predict activity in the social brain network? A genetic moderation effect," Soc Cogn Affect Neurosci, vol. 9, no. 9, pp. 1360-7, Sep, 2014.

[61] C. B. Wang, B. Wu, Y. Liu, X. H. Wu, and S. H. Han, "Challenging emotional prejudice by changing self-concept: priming independent selfconstrual reduces racial in-group bias in neural responses to other's pain," Social Cognitive and Affective Neuroscience, vol. 10, no. 9, pp. 1195-1201, Sep, 2015.

[62]Z. C. Lin, Y. Lin, and S. H. Han, "Self-construal priming modulates visual activity. underlying global/local perception," Biological Psychology, vol. 77, no. 1, pp. 93-97, Jan, 2008.

[63] J. Sui, Y. Y. Hong, C. H. Liu, G. W. Humphreys, and S. H. Han, "Dynamic cultural modulation of neural responses to one's own and friend's faces," Social Cognitive and Affective Neuroscience, vol. 8, no. 3, pp. 326-332, Mar, 2013.

[64] C. B. Wang, D. Oyserman, Q. Liu, H. Li, and S. H. Han, "Accessible cultural mind-set modulates default mode activity: Evidence for the culturally situated brain," Social Neuroscience, vol. 8, no. 3, pp. 203-216, May 1, 2013.

[65] C. Jiang, M. E. W. Varnum, Y. Y. Hou, and S. H. Han, "Distinct effects of self-construal priming on empathic neural responses in Chinese and Westerners," Social Neuroscience, vol. 9, no. 2, pp. 130-138, Mar 4, 2014.

[66] S. I. Dimitriadis, N. A. Laskaris, P. G. Simos, S. Micheloyannis, J. M. Fletcher, R. Rezaie, and A. C. Papanicolaou, "Altered temporal correlations in resting-state connectivity fluctuations in children with reading difficulties detected via MEG," Neuroimage, vol. 83, pp. 307-317, 2013.

[67] X. Shen, E. S. Finn, D. Scheinost, M. D. Rosenberg, M. M. Chun, X. Papademetris, and R. T. Constable, "Using connectome-based predictive 
modeling to predict individual behavior from brain connectivity," Nat Protoc, vol. 12, no. 3, pp. 506-518, Mar, 2017.

[68] B. A. Clementz, J. A. Sweeney, J. P. Hamm, E. I. Ivleva, L. E. Ethridge, G. D. Pearlson, M. S. Keshavan, and C. A. Tamminga, "Identification of Distinct Psychosis Biotypes Using Brain-Based Biomarkers," The American journal of psychiatry, vol. 173, no. 4, pp. 373-384, 2016/04//, 2016.

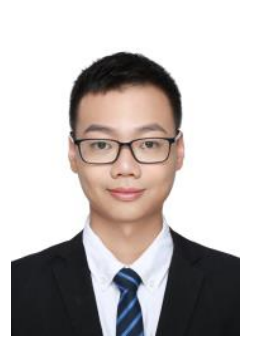

Yifan Zhu (S'19) received his Ph.D. degree from School of Computer Science \& Technology, Beijing Institute of Technology, Beijing, China, in 2021. Before that, he received the B.E. degree in computer science from Beijing Information Science \& Technology University, Beijing, China in 2016. His research interests include graph-based data mining, knowledge graph and intelligent systems.

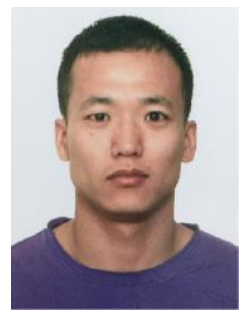

Xuesong Li received his Ph.D. degree in Center for Biomedical Imaging Research, Tsinghua University, and he is an assistant professor at Beijing Institute of Technology. His academic and clinical focus is to use advanced medical imaging techniques (sMRI, DTI, fMRI etc.), algorithms (Compressed sensing, Machine learning, Deep Learning, Big Data Analysis etc.) to understand brain connectivity, and to improve clinical practice for neurological diseases.

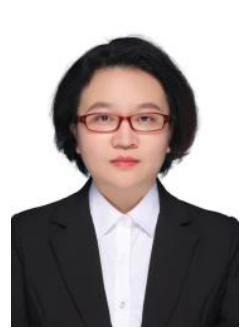

Yang Sun is an associate Professor of Shenyang Normal University. She received $\mathrm{PhD}$ in social psychology at Tsinghua University in 2017. After that, she as a postdoc, conducted social cognitive neuroscience research at the Center for Biomedical Imaging Research, Tsinghua University from 2017 to 2020 . She engaged in social and cultural cognitive neuroscience research, mainly using EEG and fMRI to explore the neural mechanism of self and systematic discussion of the self-regulation of information processing process, especially primary perceptual processing and cultural difference.

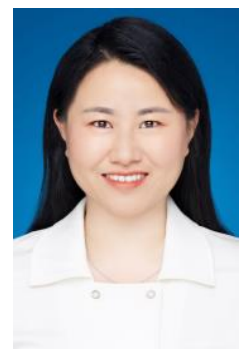

Haixu Wang received her Ph.D. degree in School of Social Sciences, Tsinghua University. Her academic focus is to use methods of experimental psychology including behavioral test, cognitive assessment, psychological scale etc. and cognitive neuroscience technologies including fMRI, VBM, ERP etc. to explore cognitive characteristics for older adults and

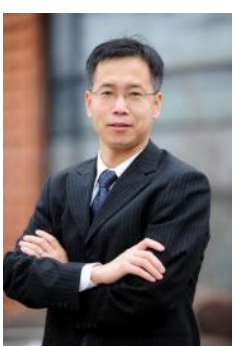

Hua Guo is a tenured associate professor at Center for Biomedical Imaging Research, Department of Biomedical Engineering at Tsinghua University in Beijing. He received his Ph.D. from the department of Biomedical Engineering at Duke University in 2006. He was on the faculty of Medical Center of New York University from 2008 to 2010 and worked on MR angiography. Before that he once worked in Siemens as a scientist and at the University of Hong Kong as a postdoc. The primary research of Dr. Guo's lab is focused on two core areas in MRI. One is MRI physics including novel sequence design and image reconstruction, which can potentially provide high spatiotemporal resolution and multi-parametric quantitative imaging. Particularly, he and his team are working on advanced diffusion weighted imaging including resolution improvement, motion correction and artifact control for different organs. The other area is the translational applications of advanced MRI techniques for accurate diagnosis and disease management. He has established substantial collaborations with hospitals in various aspects including neuroimaging, body imaging and surgery planning. Dr. Guo has been serving on the editorial board of several scientific journals and is an active reviewer for more than 10 MRI related journals.

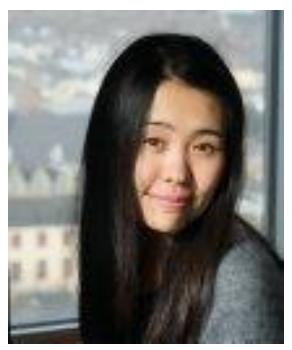

Jie Sui is a full professor in the School of Psychology at the University of Aberdeen. She received her BA in psychology joined with computer science, and then $\mathrm{PhD}$ in cognitive neuroscience at Peking University. As a principal investigator of the Social Cognition and Cultural Neuroscience Laboratory at the University of Oxford, she sought to understand the interactions among the brain, mind, and culture based on social biases (self, reward, and emotion). She was a reader of cognitive neuroscience at Bath from 2016 until she moved to Aberdeen in 2019. Her laboratory has employed fMRI, EEG, lesion-symptom mapping, mathematical models, and machine learning techniques to understand neural substrates of self biases and how aberrant self-representation leads to different mental disorders or brain disorders. Recent work has demonstrated the existence of neural circuits for driving biases to self- and reward-related stimuli operating in parallel with the neural circuits supporting cognitive control. She has served on several editorial boards and as a reviewer for numerous grants and journals.

children. 